\title{
Circ_0037866 Contributes to the Tumorigenesis of Renal Cell Carcinoma by Sequestering miR-384 to Elevate Chromobox 5 Expression
}

\author{
Xiaoqiang Shi Shichao Song Ying Gao Zhenyu Cui Wentao Wang \\ Mingkai Liu
}

Department of Urology, Affiliated Hospital of Hebei University, Baoding, China

\section{Highlights}

- Circ_0037866 is highly expressed in RCC tissues and cells.

- Knockdown of circ_0037866 suppresses RCC cell growth and mobility in vitro.

- Circ_0037866 silencing represses RCC cell proliferation and EMT in vivo.

- The circ_0037866/miR-384/CBX5 axis is identified in RCC cells.

- Circ_0037866 exerts its oncogenic role via miR-384/CBX5 axis in RCC cells.

\section{Keywords}

circ_0037866 $\cdot \mathrm{miR}-384 \cdot$ Chromobox $5 \cdot$ Renal cell

carcinoma $\cdot$ Apoptosis

\begin{abstract}
Background: Circular RNAs (circRNAs) were demonstrated to have roles in the carcinogenesis of renal cell carcinoma (RCC). Hence, this work aimed to determine the functions and molecular mechanism of circ_0037866 in regulating the progression of RCC. Methods: Quantitative real-time polymerase chain reaction and Western blotting were used to detect the levels of genes and proteins. In vitro assays, including colony formation, 5-ethynyl-2'-deoxyuridine, flow cytometry, transwell assays, and in vivo tumor formation, were conducted to investigate the effects of circ_0037866 on RCC tumorigenesis. Dual-luciferase reporter assay, RNA pull-down, and RNA immunoprecipitation assay were used toconfirm theinteraction between miR-384andcirc_0037866
\end{abstract}

Karger@karger.com www.karger.com/kbr

Karger $\stackrel{\text { ' }}{5}$

GOPEN ACCESS
(C) 2022 The Author(s)

Published by S. Karger AG, Basel

This is an Open Access article licensed under the Creative Common Attribution-NonCommercial-4.0 International License (CC BY-NC) (http://www.karger.com/Services/OpenAccessLicense), applicable to the online version of the article only. Usage and distribution for commercial purposes requires written permission. or Chromobox 5 (CBX5). Results: Circ_0037866 is a stable circRNA and was found to be increased in RCC tissues and cells. Functionally, circ_0037866 silencing suppressed RCC cell survival, invasion, and migration in vitro, and impeded RCC cell tumorigenesis in the subcutaneous xenograft model. Mechanistically, circ_0037866 could function as a sponge for miR-384 to elevate the expression of its target CBX5. Furthermore, a series of rescue experiments showed that miR384 inhibition reversed the anticancer effects of circ_0037866 knockdown on RCC cells; besides that, miR-384 restoration suppressed RCC cell growth and mobility, which were attenuated by CBX5 overexpression. Conclusion: Circ_0037866 knockdown restrains the tumorigenesis of RCC by miR-384/ $\mathrm{CBX} 5$, revealing a promising molecular target for RCC therapy.

(C) 2022 The Author(s)

Published by S. Karger AG, Basel

Xiaoqiang Shi and Shichao Song contributed equally to this work.
Correspondence to:

Mingkai Liu, liumingkai2186@163.com 


\section{Introduction}

Renal cell carcinoma (RCC) ranks as the third most common human urological cancer worldwide in 2018 [1], representing about $4 \%$ of adult malignancies [2]. Currently, surgical resection remains the most effective treatment for RCC; however, one-third of patients still eventually developed recurrence or metastasis after surgery [3, 4]. Currently, molecular targeted therapies have attracted much attention. They are revolutionized therapeutics which block cancer growth by interfering with specific molecules requested for carcinogenesis, and the Food and Drug Administration-approved molecular targeted drugs have indicated clinical success in the treatment of many types of cancers $[5,6]$. Therefore, further investigations on the molecular biology of RCC tumorigenesis are indispensable for the development of targeted drugs for RCC.

Circular RNAs (circRNAs) are a class of noncoding transcripts characterized by the covalently closed loop structure that lacks free $3^{\prime}$ or $5^{\prime}$ ends, which are highly stable and conservative compared with other RNA types and less susceptible to the regular mechanisms of linear RNA decay [7, 8]. CircRNAs exist widely across various organs and tissues and have been revealed to play pivotal roles in many cellular biological processes $[9,10]$. Recently, circRNAs were shown to be differentially expressed in diverse diseases and broadly involved in the initiation and progression of diseases including cancer in a variety of ways $[11,12]$. For example, has_circ_104348 was demonstrated to facilitate hepatocellular carcinoma tumorigenesis and metastasis by elevating RTKN2 through sequestering miR-187-3p [13]. Zhao et al. [14] showed that circRNA_MYLK silencing reduced cell proliferative rate in ovarian cancer via sponging miR-652. Circ_0037866 is a circRNA formed by the back-splicing of thioredoxin domain containing 11 (TXNDC11) gene, located at chr16: $11778020-11830089$ with a length of $1,980 \mathrm{bp}$, which was shown to be highly expressed in RCC issues [15]. Additionally, circTXNDC11 (ID: has_circ_0037866) was revealed to impede RCC cell to proliferate and invade by activating the MAPK/ERK pathway [16]. However, largescale identifications of circ_0037866 in RCC progression cells were not yet reported.

Herein, the functions of circ_0037866 in RCC cell malignant phenotypes in vitro and in vivo were investigated in this study, moreover, the mechanism by which circ_0037866 exerted its oncogenic role in RCC was further explored, which might provide a novel insight into the development of circRNA-based molecular targeted therapy.

\section{Materials and Methods}

\section{Clinical Tissue Specimens}

The RCC tissues and adjacent normal tissues were drawn from 58 RCC patients who had undergone radical nephrectomy without neoadjuvant chemotherapy or radiotherapy at Affiliated Hospital of Hebei University and immediately snap-frozen in liquid nitrogen until further analysis. Written informed consent was collected from all subjects before sample collection, and this experiment was authorized by the Ethics Committee of Affiliated Hospital of Hebei University.

\section{Cell Culture}

Human HK-2 cell line and RCC cell lines (A498, 786-O, and 769-P) were obtained from the Cell Bank of Shanghai Institute of Cell Biology (Shanghai, China). All cells were seeded in RPMI1640 medium (Invitrogen, Carlsbad, CA, USA) containing 1\% penicillin-streptomycin (Solarbio, Shanghai, China) and $10 \%$ fetal bovine serum (Invitrogen) at $37^{\circ} \mathrm{C}$ with $5 \% \mathrm{CO}_{2}$.

\section{Plasmid Transfection}

Two short hairpin RNAs (shRNAs) targeting circ_0037866 (sh-circ_0037866\#1 and sh-circ_0037866\#2), pcDNA3.1/Chromobox 5 (CBX5) overexpression plasmid, and their negative controls (sh-NC or pcDNA) were synthesized by GeneChem (Shanghai, China). The mimic or inhibitor of miR-384 and negative controls (miR-NC or anti-miR-NC) was provided by Ribobio (Guangzhou, China). Then Lipofectamine 2000 (Invitrogen) was employed to perform transient transfection in A498 and 786-O cells.

Quantitative Real-Time Polymerase Chain Reaction

Total RNA was prepared by using TRIzol reagent (Life Technologies, Carlsbad, CA, USA) and RNA concentration was qualified by a spectrophotometer. The cDNA was generated by reverse transcription with the High-Capacity cDNA Reverse Transcription Kit or TaqMan MicroRNA Reverse Transcription Kit (Life Technologies), followed by the quantitative real-time polymerase chain reaction (qRT-PCR) with TB Green ${ }^{\circledR}$ Premix Ex Taq ${ }^{\mathrm{TM}}$ Kit (Takara, Dalian, China). The CT value was detected and normalized to GAPDH or U6. Specific primer sequences are shown in Table 1 .

\section{RNase $R$ Digestion and Actinomycin D Treatment}

Total RNAs $(3 \mu \mathrm{g})$ isolated from cultured A498 and 786-O cells were incubated with $3 \mathrm{U} / \mu \mathrm{g}$ of RNase R (Sigma-Aldrich, St. Louis, MO, USA) or Mock without enzymatic activity for $1 \mathrm{~h}$ at $37^{\circ} \mathrm{C}$. Then the levels of circ_0037866 and linear TXNDC11 were measured using qRT-PCR. A498 and 786-O cells were mixed with 5 $\mu \mathrm{g} / \mathrm{mL}$ Actinomycin D for $0 \mathrm{~h}, 6 \mathrm{~h}, 12 \mathrm{~h}$, or $24 \mathrm{~h}$ to block transcription, and the half-life of circ_0037866 and linear TXNDC11 was evaluated using qRT-PCR.

\section{Colony Formation Assay}

After transfection, A498 and 786-O cells were plated in a sixwell plate and cultured for another 14 days. The medium was replaced every 3 days. The cloned cells in five fields were stained with $0.1 \%$ crystal violet (Solarbio) and calculated using the microscope. 


\section{5-Ethynyl-2'-Deoxyuridine Assay}

A498 and 786-O cells after assigned transfection were seeded onto a 96-well plate with $10 \mu \mathrm{L}$ of 5-ethynyl-2'-deoxyuridine (EdU) reagent (RiboBio) in $90 \mu \mathrm{L}$ of fresh culture medium and then incubated for another $2 \mathrm{~h}$. After being fixed and permeabilized, cells were stained with Apollo reaction mixture for $30 \mathrm{~min}$ away from light. Cell nucleus was stained with DPAI. Later, EdUpositive cells were determined by using a fluorescence microscope.

\section{Flow Cytometer}

Transfected A498 and 786-O cells were washed with PBS and trypsin to obtain the single-cell suspensions. After being fixed in ice-cold $70 \%$ ethanol, cells were mixed with $5 \mu \mathrm{L}$ FITC-AnnexinV and $5 \mu \mathrm{L}$ propidium iodide (PI) (Sigma-Aldrich) for $20 \mathrm{~min}$ in the dark. Apoptosis was detected by FACScan flow cytometry (BD Biosciences, San Diego, CA, USA).

\section{Transwell Assay}

Transwell inserts in 24 wells (Costar, Corning, Switzerland) with an $8-\mu \mathrm{m}$ pore membrane or a Matrigel-coated membrane (BD Biosciences) were used to investigate cell migration and invasion abilities. The upper chamber containing $200 \mu \mathrm{L}$ serum-free medium was inoculated with transfected A498 and 786-O cells, and $500 \mu \mathrm{L}$ of complete culture medium was filled into the lower chamber. Cells on the membranes of the lower compartment were counted $24 \mathrm{~h}$ later.

\section{Western Blotting}

Total proteins were extracted by RIPA lysis buffer (Beyotime, Beijing, China). After qualification by a BCA method, proteins were loaded onto $10 \%$ SDS-polyacrylamide gel electrophoresis for separating and then transferred to a polyvinylidene fluoride (PVDF) membrane (Millipore, Darmstadt, Germany). Subsequently, the membrane was incubated with primary antibodies against PCNA (1:5,000, ab29), Bcl-2 (1:1,000, ab194583), matrix metallopeptidase9 (MMP9) (1:2,000, ab38898), Cleaved-caspase 3 $(1: 2,000, \mathrm{ab} 2302)$, and GAPDH $(1: 5,000, \mathrm{ab} 181602)$ at $4^{\circ} \mathrm{C}$ overnight, and then probed with a secondary antibody (dilution $1: 1,000)$ for $1 \mathrm{~h}$ at room temperature. Protein bands were studied using the ECL system (Solarbio). All antibodies were obtained from Abcam (Cambridge, MA, USA).

\section{Tumor Xenograft Assay}

$\mathrm{BALB} / \mathrm{c}$ nude mice (3-4 weeks old, $N=12$ ) were purchased from Charles River Labs (Beijing, China). Lentivirus vectors carryingsh-circ_0037866\#1 orsh-NCwere constructed by GeneChem, and then transfected into 786-O cells. The stably transfected 786-O cells were selected by puromycin and then $1 \times 10^{6}$ cells were subcutaneously injected into the right flank of two blindly randomized nude mice groups ( $N=6$ per group). Tumor size was measured every week with a Vernier caliper, and tumor volume was calculated using the following formula: volume $=\left(\right.$ length $\times$ width $\left.{ }^{2}\right) / 2$. All mice were killed, and the tumors were excised 4 weeks later, weighed, and fixed in formalin for immunohistochemistry analysis with Ki-67 and Vimentin antibodies as described previously [17].

\section{RNA Pull-Down Assay}

Biotinylated circ_0037866 probes or the control probes (BioNC) were synthesized by Genepharma (Shanghai, China). These
Table 1. Primers sequences used for qRT-PCR

\begin{tabular}{|c|c|}
\hline Name & Primers for qRT-PCR ( $\left.5^{\prime}-3^{\prime}\right)$ \\
\hline \multicolumn{2}{|l|}{ circ_0037866 } \\
\hline Forward & ACACATTCACTGTGGCAAGGA \\
\hline Reverse & AAAGCTGACAGGTGGCTITG \\
\hline \multicolumn{2}{|l|}{ TXNDC11 } \\
\hline Forward & ACGTTCGACGGGATTCAGAG \\
\hline Reverse & GCACTCATGGGGCCTTTGTA \\
\hline \multicolumn{2}{|l|}{ miR-384 } \\
\hline Forward & GCGGCATTCCTAGAAATTGTT \\
\hline Reverse & CTCAACTGGTGTCGTGGAGT \\
\hline \multicolumn{2}{|l|}{$\operatorname{miR}-665$} \\
\hline Forward & TGCTACCAGGAGGCTGAG \\
\hline Reverse & СTCAACTGGTGTCGTGGAGT \\
\hline \multicolumn{2}{|l|}{ miR-331-3p } \\
\hline Forward & GTATGAGCCCCTGGGCCTAT \\
\hline Reverse & CTCAACTGGTGTCGTGGA \\
\hline \multicolumn{2}{|l|}{ CBX5 } \\
\hline Forward & GATATCGCTCGGGGCTITGA \\
\hline Reverse & TGCAAGAACCAGGTCAGCTT \\
\hline \multicolumn{2}{|l|}{ GAPDH } \\
\hline Forward & GACAGTCAGCCGCATCTTCT \\
\hline Reverse & GCGCCCAATACGACCAAATC \\
\hline \multicolumn{2}{|l|}{ U6 } \\
\hline Forward & СTCGCTTCGGCAGCACA \\
\hline Reverse & AACGCTTCACGAATITGCGT \\
\hline
\end{tabular}

RNA probes were incubated with the lysates of A498 and 786-O cells, followed by interaction with streptavidin-coupled magnetic beads as per the instructions of Pierce ${ }^{\mathrm{TM}}$ Magnetic RNA PullDown Kit (Rockford, IL, USA). The pull-down product was then analyzed by qRT-PCR after purification by TRIzol.

\section{Dual-Luciferase Reporter Assay}

The fragments of circ_0037866 and CBX5 3'UTR covering the target sites in miR-384 and point mutated (mut) sequences in putative binding sites generated by GeneChem were amplified and inserted into the pGL3-Basic vector (Promega, Madison, WI, USA) to generate wild-type (wt) and mut luciferase reporter vectors (pGL3-wt-circ_0037866/CBX5 or pGL3-mut-circ_0037866/ CBX5). A498 and 786-O cells in 24-well plates were cotransfected with constructed plasmids, pRL-TK Renilla vector, and miR-384 or miR-NC, and luciferase activity was detected after $48 \mathrm{~h}$ of transfection.

\section{RNA Immunoprecipitation Assay}

A498 and 786-O cells were lysed in RNA immunoprecipitation lysis buffer (Millipore) and then incubated with magnetic beads conjugated with IgG or AGO2 antibodies at $4^{\circ} \mathrm{C}$ overnight. The bead/antibody/lysate mixture was treated with proteinase $\mathrm{K}$, and the enrichment of circ_0037866 with AGO immunoprecipitation was calculated using qRT-PCR after isolation with TRIzol.

Statistical Analyses

The results in the bar graphs were expressed as mean \pm standard deviation. Student's $t$ test was used for the comparison of da- 


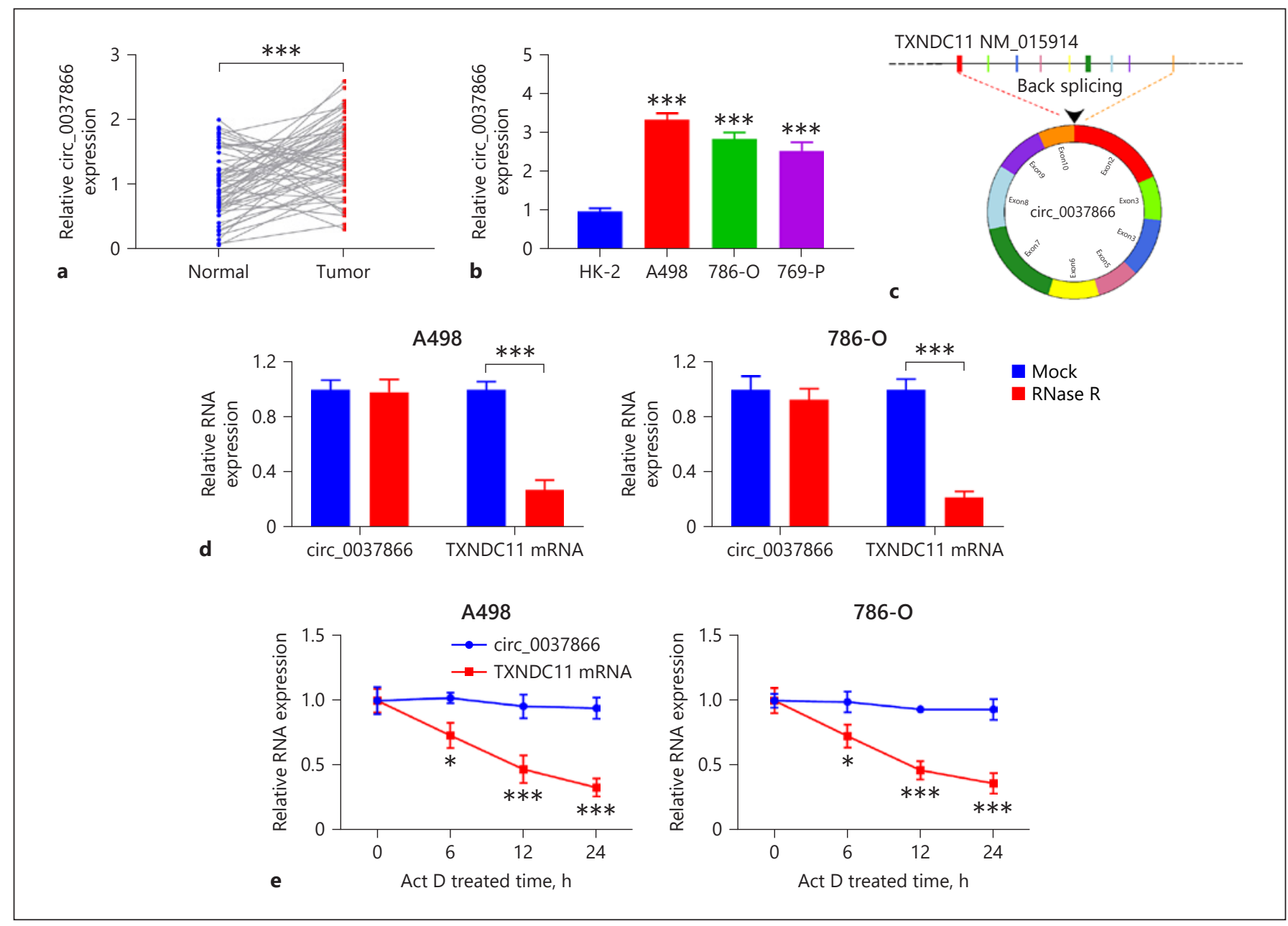

Fig. 1. Circ_0037866 is highly expressed in RCC tissues and cells. a, b qRT-PCR analysis of circ_0037866 expression in RCC tissues and adjacent normal tissues, as well as in RCC cells and normal HK-2 cells. c Schematic diagram of the genomic location and formation of circ_0037866.d A498 and 786-O cells were treated with
RNase R or Mock and the levels of circ_0037866 and linear TXNDC11 were analyzed by qRT-PCR. e The expression of circ_0037866 and linear TXNDC11 was detected by qRT-PCR in A498 and 786-O cells after Actinomycin D treatment. ${ }^{*} p<0.05$, $* * * p<0.001$. tasets containing two groups. Statistical difference among multiple groups was determined by analysis of variance. The expression correlation was analyzed using Pearson's correlation coefficient assay. $p<0.05$ indicates statistical differences $\left({ }^{*} p<0.05,{ }^{* *} p<0.01\right.$, $* * * p<0.001)$.

\section{Results}

Circ_0037866 Is Highly Expressed in RCC Tissues and Cells

We first detected the expression profile of circ_0037866 in 58 clinical RCC samples. The results of qRT-PCR showed that circ_0037866 expression was higher in RCC tissues than in normal tissues (Fig. 1a). Subsequently, the same elevation results of circ_0037866 expression were observed in RCC cell lines compared with the normal HK-2 cells (Fig. 1b). Circ_0037866 is formed by backsplicing of the exon $2-10$ of the TXNDC11 gene (Fig. 1c). Then, the circular characteristics of circ_0037866 were analyzed. We found that circ_0037866 could resist the degradation by RNase $\mathrm{R}$ relative to the linear TXNDC11 in A498 and 786-O cells (Fig. 1d). Moreover, Actinomycin D treatment showed that the half-life of circ_0037866 exceeded $24 \mathrm{~h}$, while that of linear TXNDC11 mRNA was about $6 \mathrm{~h}$ in A498 and 786-O cells (Fig. 1e). Therefore, these data indicated that circ_0037866 was an abundant circRNA in RCC, and might be associated with the progression of RCC. 


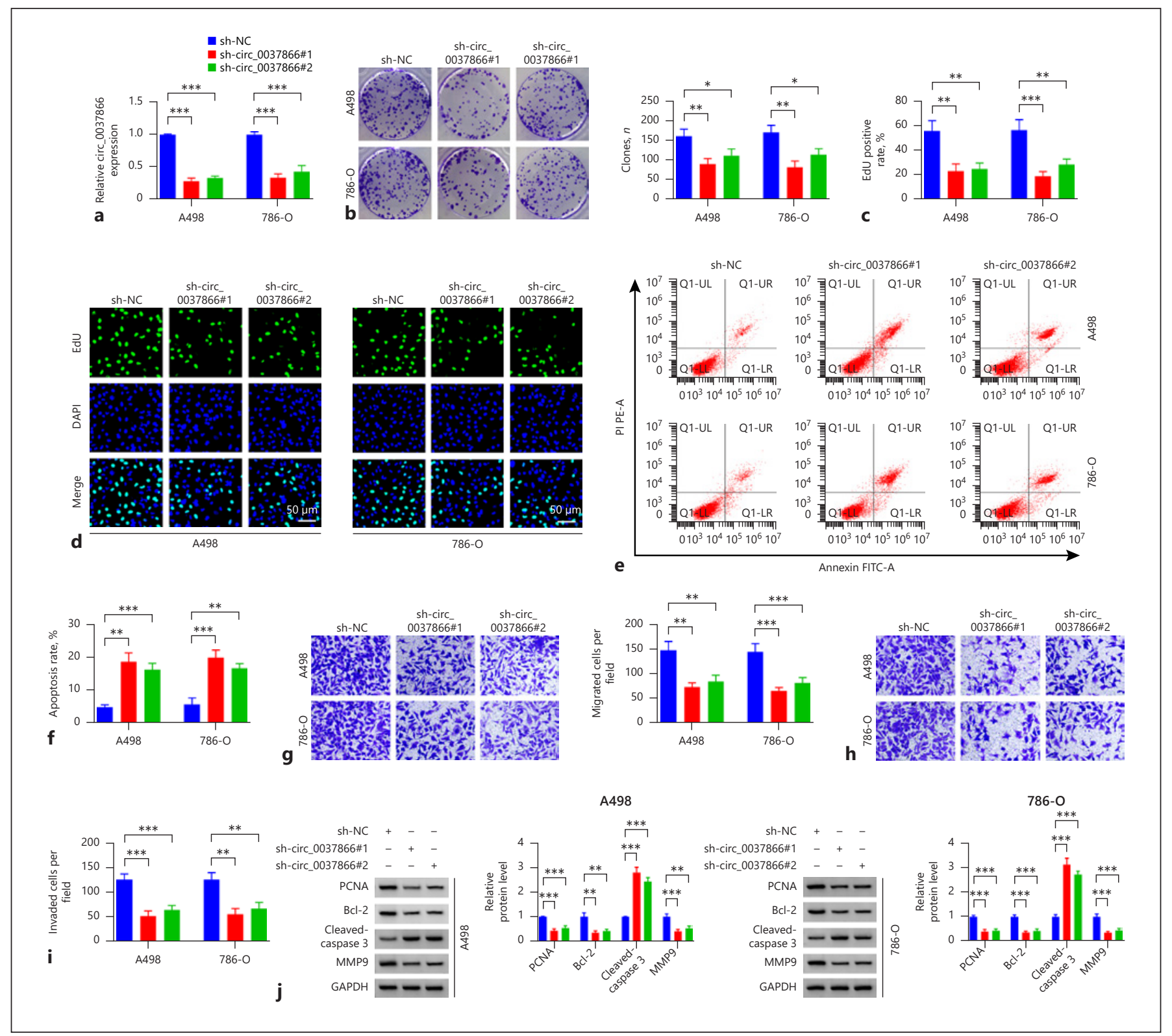

Fig. 2. Knockdown of circ_0037866 suppresses RCC cell growth, migration, and invasion in vitro. a-j A498 and 786-O cells were transfected with sh-circ_0037866\#1, sh-circ_0037866\#2, or sh-NC. a The expression level of circ_0037866 was detected by qRT-PCR after transfection. b-d Cell transfection analysis using colony formation and EdU assays. e, f Flow cytometry for cell apoptosis. $\mathbf{g}-\mathbf{i}$ Transwell assay for cell migration and invasion abilities. j Western blotting analysis for the protein levels of PCNA, Bcl-2, Cleaved-caspase 3, and MMP9. ${ }^{*} p<0.05$, ${ }^{* *} p<0.01,{ }^{* * *} p<0.001$.

Knockdown of circ_0037866 Suppresses RCC Cell Growth, Migration, and Invasion in vitro

To probe the biological function of circ_0037866 in RCC cells, two shRNAs targeting circ_0037866 were designed to silence circ_0037866 in A498 and 786-O cells, as expected, circ_0037866 expression was significantly reduced after either sh-circ_0037866\#1 or shcirc_0037866\#2 introduction compared with sh-NC transfection (Fig. 2a). Thereafter, colony formation and EdU assays showed that circ_0037866 knockdown sup- 


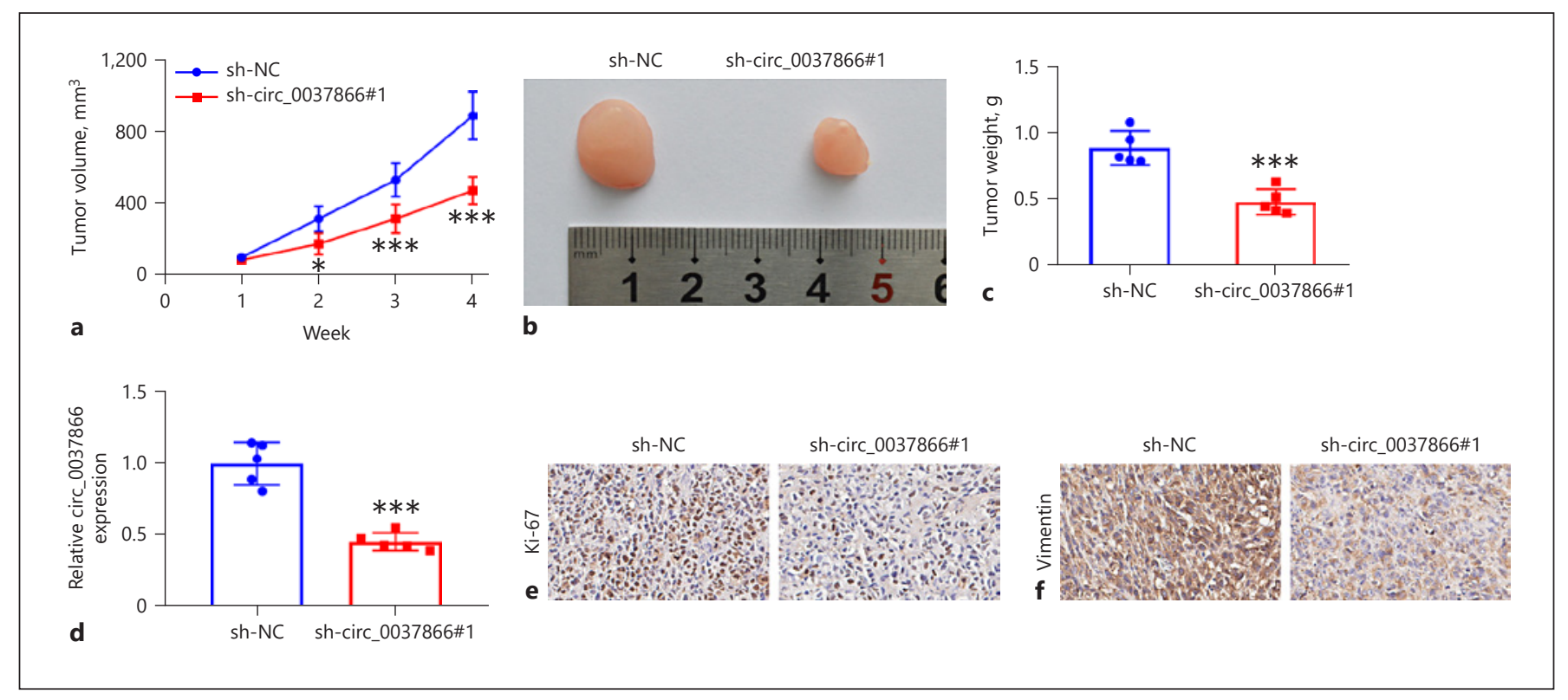

Fig. 3. Silencing of circ_0037866 impedes RCC tumor tumorigenesis in vivo. a Tumor volume was measured once a week and the growth curves were drawn. $\mathbf{b}$ The representative images of xenograft tumors are displayed. c Tumor weight of xenograft tumors was detected. $\mathbf{d}$ qRT-PCR analysis of circ_0037866 expression levels in tumor masses from each group. e, f IHC staining for Ki-67 and Vimentin protein in tumor masses from each group. $* * * p<0.001$. IHC, immunohistochemistry.

pressed cell cloning capability and DNA synthesis activity in A498 and 786-O cells (Fig. 2b-d). Conversely, the apoptosis rates of A498 and 786-O cells were overtly reinforced by circ_0037866 knockdown (Fig. 2e, f). Meanwhile, transwell assay showed that circ_0037866 silencing inhibited A498 and 786-O cell migration and invasion abilities (Fig. 2g-i). Furthermore, the related markers about the above behaviors were detected in A498 and 786-O cells, the results of Western blotting showed that circ_0037866 deletion led to the decreases in PCNA, Bcl2, and MMP9 proteins and the increase in Cleaved-caspase 3 protein (Fig. 2j). Taken together, circ_0037866 knockdown partly repressed RCC cell tumorigenesis.

\section{Silencing of circ_0037866 Impedes RCC Tumor}

\section{Tumorigenesis in vivo}

To clarify the clinical value of circ_0037866 on tumor growth, a subcutaneous xenograft model was established by inoculating $786-\mathrm{O}$ cells infected with lentiviruses expressing circ_0037866 shRNA and their controls into nude mice. The results suggested that the tumor volume and weight in the circ_0037866-decreased group were obviously less than that in the control group, implying the inhibition of tumor growth (Fig. 3a-c). The level of circ_0037866wasdecreasedinthetumorsofcirc_0037866- decreased group (Fig. 3d). Moreover, immunohistochemistry staining displayed that downregulation of circ_0037866 could reduce the expressions of Ki-67 and Vimentin in xenograft tumor tissues (Fig. 3e, f), suggesting the inhibition of proliferation and EMT. Collectively, circ_0037866 decrease suppressed the tumorigenesis of RCC in vivo.

\section{Circ_0037866 Is a Sponge for miR-384 in RCC Cells}

To explore the molecular mechanism underlying circ_0037866, the potential targets of circ_0037866 were predicted using bioinformatics analysis based on the Circinteractome, circBank, and starBase databases, among which 3 miRNAs were selected from the overlap between the databases (Fig. 4a). Then RNA pull-down assay in A498 and 786-O cells showed that miR-384 was significantly captured by the biotinylated circ_0037866 probe with markedly enhanced fold changes (Fig. 4b, c). The putative conserved target site of miR-384 on circ_0037866 is shown in Figure 4d. After confirming the elevation efficiency of miR-384 mimic (Fig. 4e), the dual-luciferase reporter assay was performed, we discovered that miR-384 mimic significantly reduced the luciferase activity of the wt circ_0067582 pGL3 reporter, but failed to affect the mut one in A498 and 786-O 


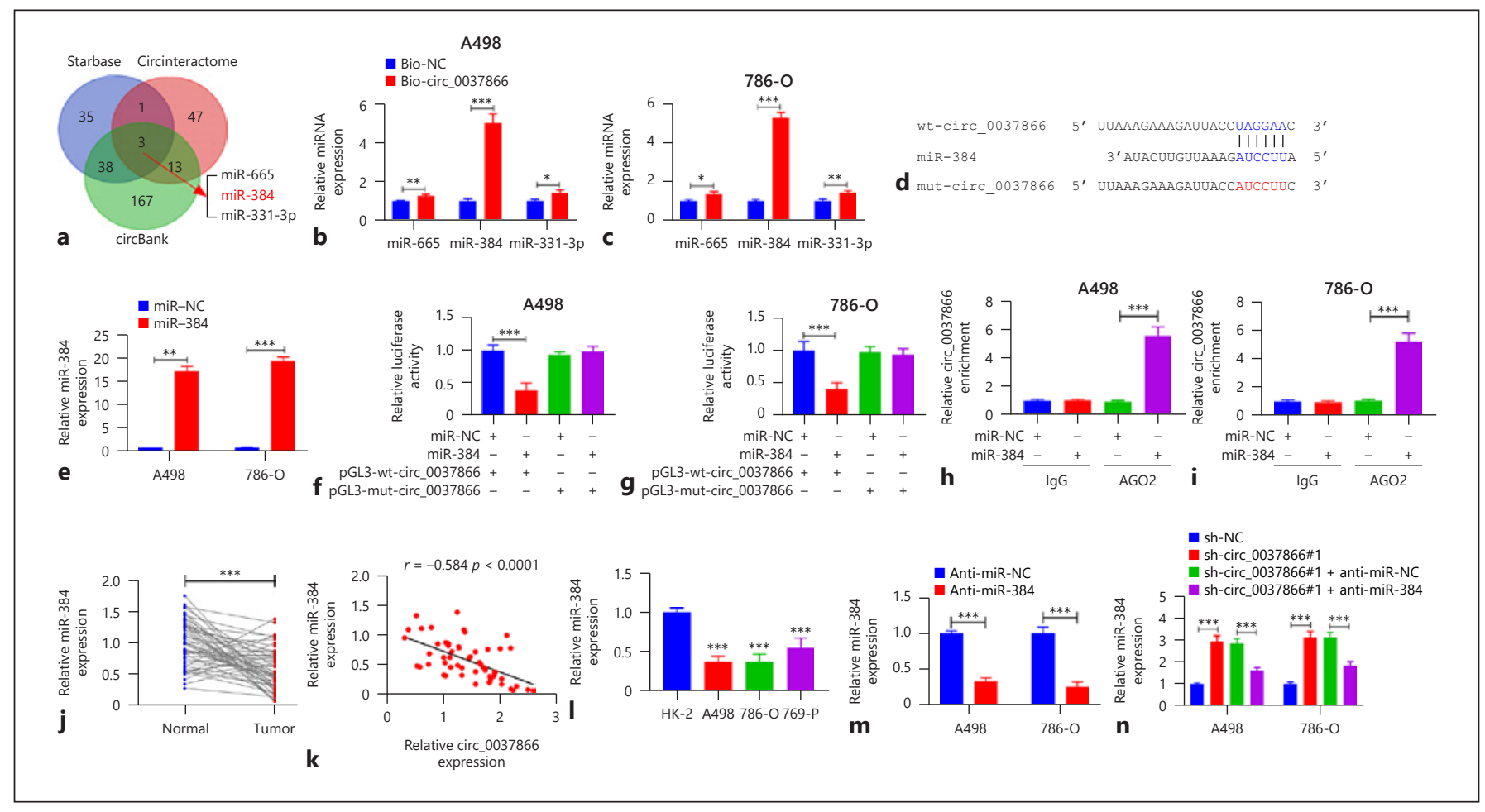

Fig. 4. Circ_0037866 is a sponge for miR-384 in RCC cells. a Schematic illustration exhibiting overlapping of the target miRNAs of circ_0037866 predicted by Circinteractome, circBank, and starBase databases. b, c RNA pull-down with a biotinylated circ_0037866 probe was conducted in A498 and 786-O cells and the enrichment of miR-665, miR-384, and miR-331-3p was analyzed by qRT-PCR. $\mathbf{d}$ The putative conserved target site of miR-384 on circ_0037866. e The elevation efficiency of miR-384 mimic or the control in A498 and 786-O cells was confirmed using qRTPCR. f, $\mathbf{g}$ Dual-luciferase reporter assay for the luciferase activity of wt and mut circ_0037866 reporter after miR-384 overexpression in A498 and 786-O cells. $\mathbf{h}, \mathbf{i}$ RIP assay was executed in A498

cells (Fig. 4f, g). Moreover, RNA immunoprecipitation assay showed that circ_0067582 was effectively pulled down using anti-AGO2 antibody and was highly enriched in cells transfected with miR-384 mimic relative to the controls (Fig. 4h, i), further confirming the binding of circ_0067582 with miR-384. The expression of miR-384 was found to be decreased in RCC tissues (Fig. 4j), which was negatively correlated with circ_0067582 expression (Fig. 4k). Also, its expression was downregulated in RCC cells compared with normal HK-2 cells (Fig. 4l). The results of qRT-PCR showed that miR-384 expression was decreased by miR-384 inhibitor compared with the control in A498 and 786-O cells (Fig. 4m), then it was observed that knockdown of circ_0067582 resulted in an increase in miR-384 ex- and 786-O cells after transfection with miR-384 mimic or miRNC, and levels of circ_0037866 were examined by qRT-PCR. $\mathbf{j}$ qRT-PCR analysis of miR-384 expression in RCC tissues and adjacent normal tissues. $\mathbf{k}$ miR-384 expression was negatively correlated with circ_0037866 expression in RCC tissues. I qRT-PCR analysis of miR-384 expression in RCC cells and normal HK-2 cells. $\mathbf{m}$ The knockdown efficiency of miR-384 inhibitor or antimiR-NC was validated by qRT-PCR. $\mathbf{n}$ qRT-PCR analysis of miR384 expression in A498 and 786-O cells transfected with sh-NC, sh-circ_0037866\#1, sh-circ_0037866\#1 + anti-miR-NC, or shcirc_0037866\#1+anti-miR-384. ${ }^{*} p<0.05,{ }^{* *} p<0.01,{ }^{* * *} p<0.001$.

pression, while the addition of miR-384 inhibitor reversed this elevation in A498 and 786-O cells (Fig. 4n). These results confirmed that circ_0067582 could function as a sponge for miR-384 in RCC.

\section{Knockdown of circ_0037866 Suppresses RCC Cell Growth, Migration, and Invasion by Targeting miR-384}

Subsequently, we further investigated whether circ_0037866 plays its biological role via miR-384. The results revealed that downregulation of miR-384 markedly attenuated the proliferation arrest (Fig. 5a, b), apoptosis enhancement (Fig. 5c), as well as migration and invasionsuppression(Fig.5d-f) mediated bycirc_0037866 downregulation in A498 and 786-O cells. Besides that, 


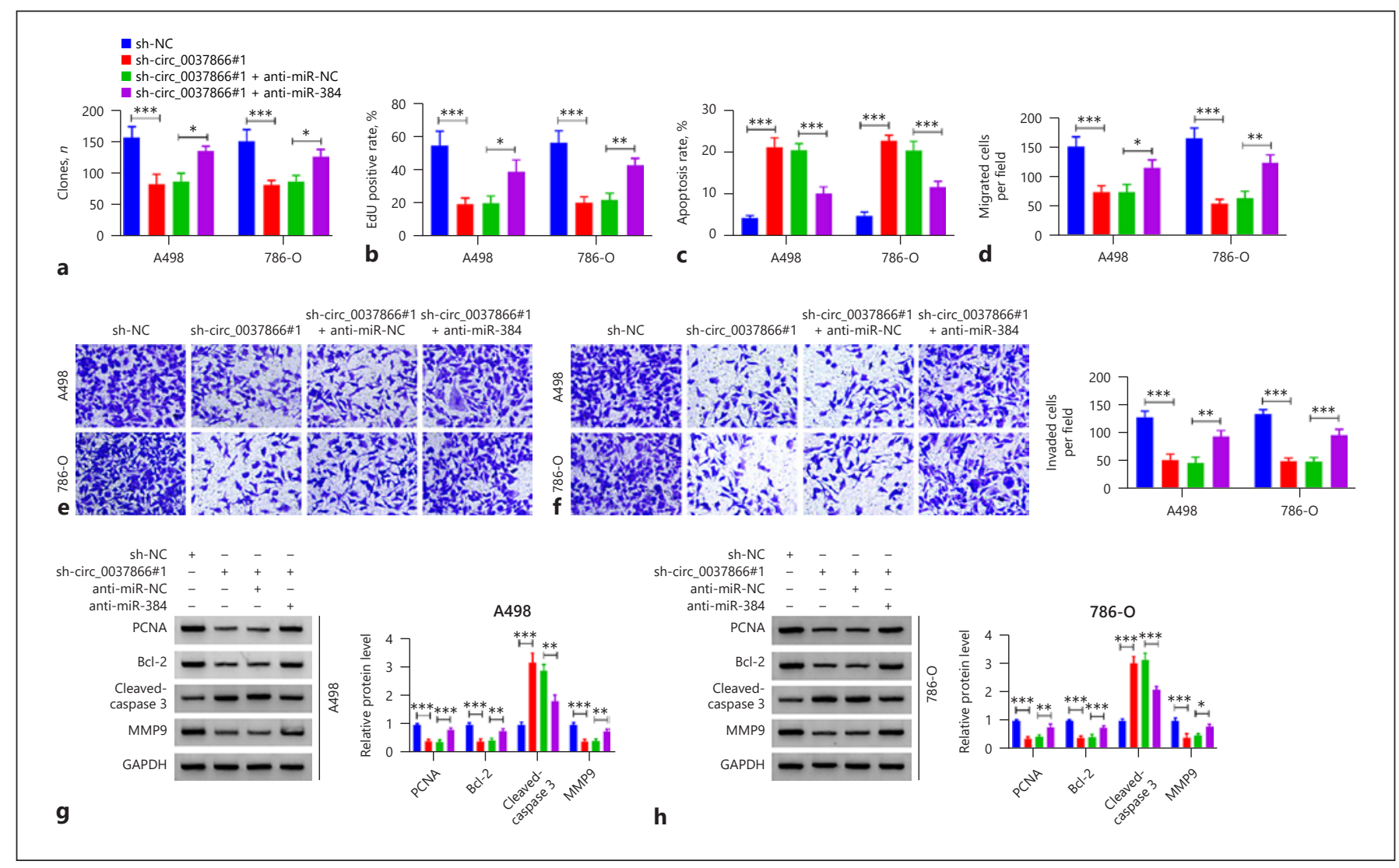

Fig. 5. Knockdown of circ_0037866 suppresses RCC cell growth, migration, and invasion by targeting miR-384. a-h A498 and 786-O cells were transfected with sh-NC, sh-circ_0037866\#1, sh-circ_0037866\#1 + anti-miR-NC, or sh-circ_0037866\#1 + anti-miR-384. a, b Cell transfection analysis using colony formation and EdU assays. c Flow cytometry for cell apoptosis. d-f Transwell assay for cell migration and invasion abilities. $\mathbf{g}$, $\mathbf{h}$ Western blotting analysis for the protein levels of PCNA, Bcl-2, Cleaved-caspase 3, and MMP9. ${ }^{*} p<0.05,{ }^{* *} p<0.01,{ }^{* * *} p<$ 0.001 .

Western blotting analysis showed that miR-384 inhibition led to the increase in PCNA, Bcl-2 and MMP9 proteins and the decrease in Cleaved-caspase 3 protein in circ_0037866-downregulated A498 and 786-O cells (Fig. 5g, h). Collectively, these results demonstrated that circ_0037866 acted as s sponge for miR-384 to regulate the tumorigenesis of RCC cells.

\section{CBX5 Is a Target of miR-384 in RCC Cells, and}

circ_0037866 Regulates CBX5 via Sponging miR-384

Next, the possible targets of miR-384 were searched through starBase database, the data displayed that CBX5 contained the conserved target site of miR-384 (Fig. 6a). As expected, the binding of miR-384 and CBX5 was then confirmed by dual-luciferase reporter assay with the decreased luciferase activity in A498 and 786-O cells co- transfected with wt CBX5 pGL3 reporter and miR-384 mimic (Fig. 6b, c). In addition, CBX5 expression was decreased by miR-384 mimic but increased by miR-384 inhibitor in A498 and 786-O cells (Fig. 6d). All these results confirmed that miR-384 negatively regulated CBX5 expression in a targeted manner.

Besides that, circ_0037866 knockdown was found to lead to a decrease in CBX5 expression in A498 and 786-O cells, which was rescued by miR-384 inhibition (Fig. 6e). Moreover, Western blotting analysis showed that transfection of CBX5 overexpression plasmids remarkably elevated CBX5 expression compared with the control (Fig. 6f), subsequently, we discovered that CBX5 transfection rescued miR-384 mimic-induced reduction of CBX5 expression level in A498 and 786-O cells (Fig. 6g). Meanwhile, CBX5 was observed to be increased in RCC tissues 


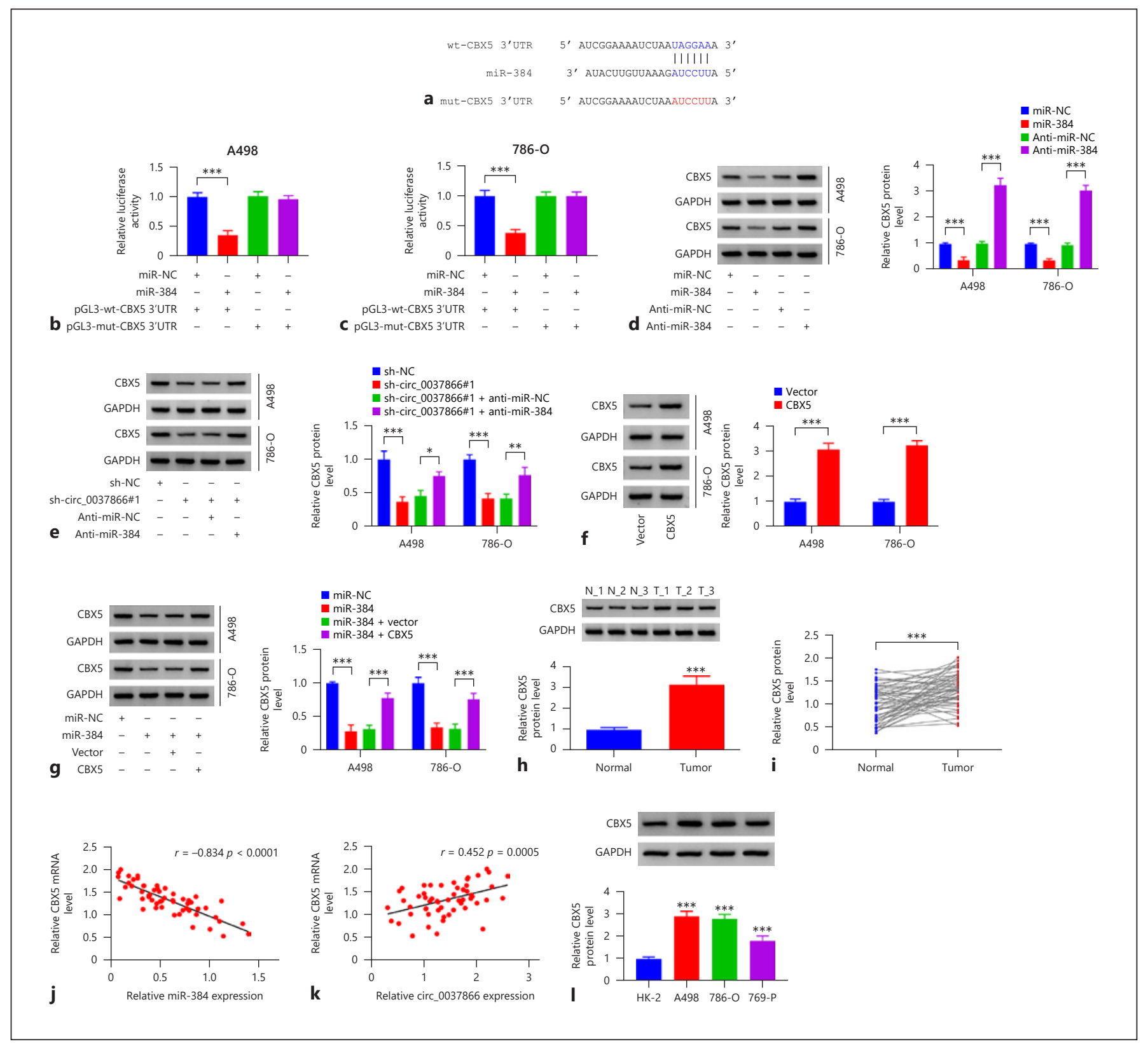

Fig. 6. CBX5 is a target of miR-384 in RCC cells, and circ_0037866 regulates CBX5 via sponging miR-384. a The conserved target site of miR-384 on CBX5. b, c Dual-luciferase reporter assay for the luciferase activity of wt and mut CBX5 reporter after miR-384 overexpression in A498 and 786-O cells. d Western blotting analysis for the level of CBX5 in miR-384 increased or decreased A498 and 786-O cells. e Western blotting analysis for CBX5 expression level in A498 and 786-O cells transfected with sh-NC, shcirc_0037866\#1, sh-circ_0037866\#1 + anti-miR-NC, or shcirc_0037866\#1 + anti-miR-384. f The elevation efficiency of
CBX5 overexpression plasmids or the control was conformed using Western blotting in A498 and 786-O cells. g Western blotting analysis for CBX5 expression level in A498 and 786-O cells transfected with miR-NC, miR-384, miR-384 + Vector, or miR-384 + CBX5. h, i Western blotting and qRT-PCR analysis of CBX5 expression in RCC tissues and adjacent normal tissues. j, k CBX5 expression was negatively correlated with miR-384 expression, but positively correlated with circ_0037866 expression in RCC tissues. I Western blotting analysis of CBX5 expression in RCC cells and normal HK-2 cells. ${ }^{*} p<0.05,{ }^{* *} p<0.01,{ }^{* * *} p<0.001$. 

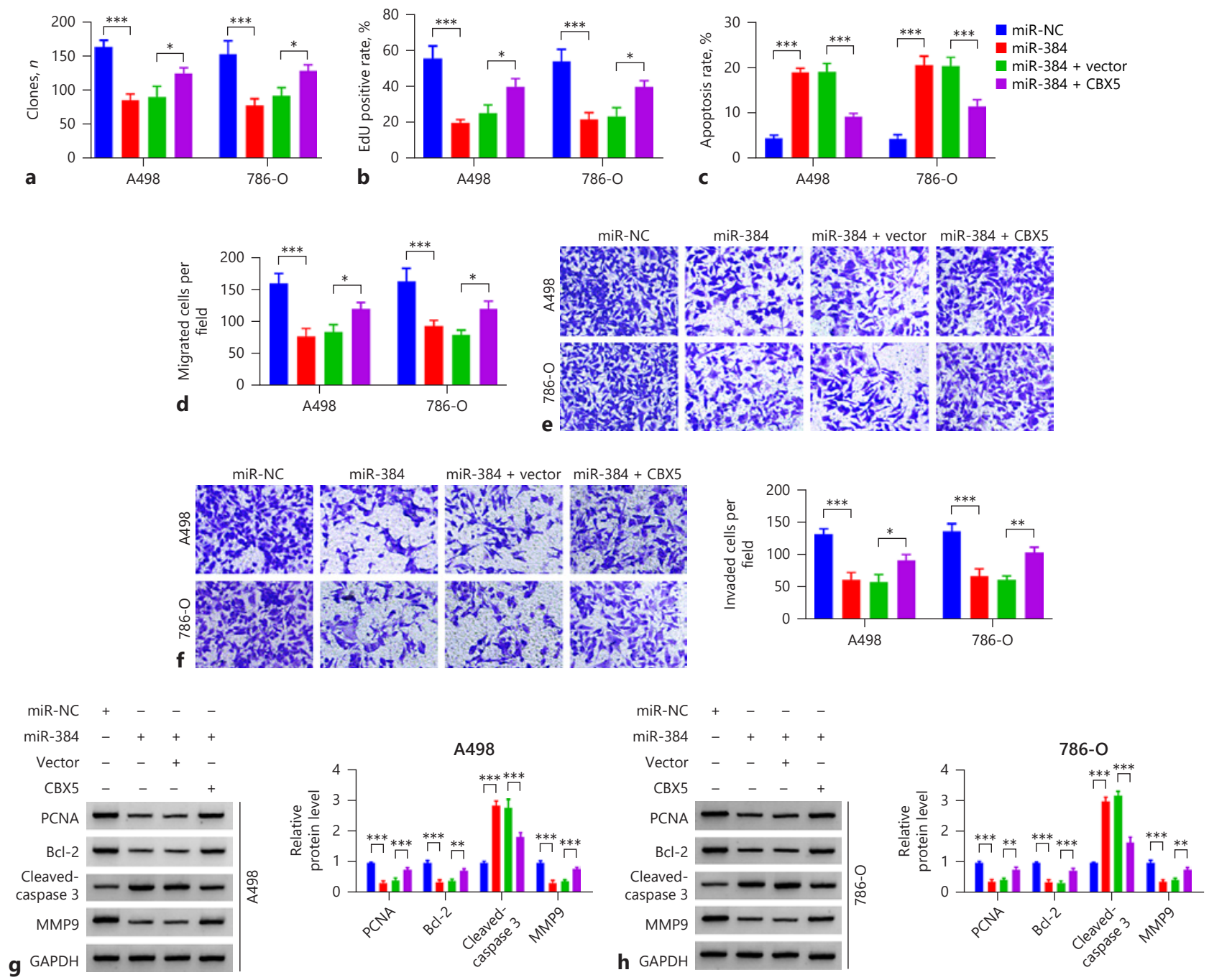

Fig. 7. MiR-384 restrains RCC cell growth, migration, and invasion by targeting CBX5. a-h A498 and 786-O cells were transfected with miR-NC, miR-384, miR-384 + Vector, or miR-384 + CBX5. a, b Cell transfection analysis using colony formation and EdU assays. c Flow cytometry for cell apoptosis. $\mathbf{d}-\mathbf{f}$ Transwell assay for cell migration and invasion abilities. $\mathbf{g}, \mathbf{h}$ Western blotting analysis for the protein levels of PCNA, Bcl-2, Cleaved-caspase 3, and MMP9. ${ }^{*} p<0.05,{ }^{* *} p<0.01,{ }^{* * *} p<0.001$.

at both the protein and mRNA levels (Fig. 6h, i), which was negatively correlated with miR-384 expression but positively correlated with circ_0037866 expression (Fig. 6j, k). Moreover, an increase in CBX5 expression was also discovered in RCC cells (Fig. 61). In all, these results identified the circ_0037866/miR-384/CBX5 axis in RCC.
MiR-384 Restrains RCC Cell Growth, Migration, and Invasion by Targeting CBX5

To probe whether miR-384/CBX5 axis had functions in RCC tumorigenesis, a series of rescue experiments were implemented. Functionally, ectopic expression of miR-384 markedly suppressed cell proliferation (Fig. 7a, b), induced cell apoptosis (Fig. 7c), and reduced cell migration and invasion abilities (Fig. 7d-f) in A498 and 786- 
O cells, which were attenuated by CBX5 up-regulation. Besides that, CBX5 overexpression could counteract the inhibitory impacts of PCNA, Bcl-2, and MMP9 protein levels and promote the effect of Cleaved-caspase 3 protein level in A498 and 786-O cells (Fig. 7g, h). Collectively, miR-384 could suppress RCC tumorigenesis by targeting CBX5.

\section{Discussion}

In recent years, circRNAs have attracted increasing research attention for their diverse impacts on the occurrence and development of RCC. Growing evidence hints at a significant role for the expression alterations of circRNAs in biological characteristics of RCC $[18,19]$. For instance, circTLK1 functioned as an oncogene to expedite the metastasis and growth in RCC by modulating the miR-495-3p/CBL axis [20]. Besides, circ-ITCH was demonstrated to perform anticancer effects in ccRCC by suppressing cell invasion and migration through the miR106b-5p/PDCD4 axis [21]. Cheng et al. [22] indicated circ_RPL23A overexpression led to cell cycle, proliferation, and metastasis arrest in ccRCC by upregulating ACAT2 through abolishing miR-1233. All the findings imply the emerging role of circRNA as a biomarker in RCC. In this work, an increased circ_0037866 expression was observed in RCC, which was consistent with previous studies [15, 16]. Moreover, silencing of circ_0037866 suppressed RCC cell proliferative, invasive, and migratory capacities, but induced cell apoptosis in vitro, besides that, circ_0037866 deletion suppressed RCC cell tumorigenesis in the mouse xenograft model. All these data confirmed the oncogenic role of circ_0037866 in RCC growth.

Accumulating evidence suggested that circRNAs can act as endogenous competitive RNAs to sponge miRNAs to regulate their biological functions, thereby regulating the expression and function of their downstream target genes $[23,24]$. Moreover, the circRNA/miRNA/mRNA regulatory network has been identified to serve a significant role in the tumorigenesis of RCC [25]. In the current review, we verified that circ_0037866 directly sponged miR-384, which targeted CBX5. Besides, circ_0037866 could indirectly regulate CBX5 expression via interacting with miR-384 in RCC cells. MiR-384 is a well-recognized tumor suppressor in many cancers $[26,27]$. In RCC, it was also demonstrated to suppress cell growth and motility [28-30], and the same results were also observed in this work. This study showed a decreased level of miR384 in RCC, moreover, rescue experiments showed that
miR-384 inhibition reversed the inhibitory action of circ_0037866 knockdown in RCC cell malignant phenotypes. CBX5, also known as HP1a, belongs to the CBX protein family, which is an important component of epigenetic regulation complexes and is involved in the progression of multiple malignancies through the blockage of differentiation and promotion of proliferation [31]. $\mathrm{Wu}$ and Zhang [32] revealed that silencing of CBX5 could block cell migration and proliferation RCC by LOXL1AS1/miR-589-5p/CBX5 axis, also indicating the carcinogenic function in RCC. In the present review, we also found an increase in CBX5 expression in RCC, thereafter, a series of rescue assays revealed that $\mathrm{CBX} 5$ up-regulation attenuated the anticancer effects of miR-384 in RCC cells. In conclusion, this work demonstrated that circ_0037866 elicited a tumor-facilitating action in RCC via upregulating CBX5 expression by abolishing miR-384, suggesting a new therapeutic biomarker for RCC-targeted therapy.

\section{Statement of Ethics}

Written informed consents were obtained from all participants and this study was permitted by the Ethics Committee of Affiliated Hospital of Hebei University and the number is 2019XA107.

\section{Conflict of Interest Statement}

The authors declare that they have no conflicts of interest.

\section{Funding Sources}

This study is not funded by any organization.

\section{Author Contributions}

All authors were involved in the management of the patients, in the conception of the manuscript, and read and approved the final manuscript. Xiaoqiang Shi and Shichao Song were involved in the drafting of the manuscript and its critical revision for important intellectual content. Ying Gao and Zhenyu Cui were involved in data collection. Wentao Wang and Mingkai Liu were involved in analysis and interpretation.

\section{Data Availability Statement}

The datasets used and/or analyzed during the present study are available from the corresponding author on reasonable request. 


\section{References}

1 Siegel RL, Miller KD, Jemal A. Cancer statistics, 2018. CA Cancer J Clin. 2018;68(1):7-30.

2 Siegel RL, Miller KD, Jemal A. Cancer statistics, 2019. CA Cancer J Clin. 2019;69(1):7-34.

3 Ljungberg B, Bensalah K, Canfield S, Dabestani S, Hofmann F, Hora M, et al. EAU guidelines on renal cell carcinoma: 2014 update. Eur Urol. 2015;67(5):913-24.

4 Chen C, Xue S, Zhang J, Chen W, Gong D, Zheng J, et al. DNA-methylation-mediated repression of miR-766-3p promotes cell proliferation via targeting SF2 expression in renal cell carcinoma. Int J Cancer. 2017;141(9): 1867-78.

5 Lee YT, Tan YJ, Oon CE. Molecular targeted therapy: treating cancer with specificity. Eur J Pharmacol. 2018;834:188-96.

6 Pérez-Herrero E, Fernández-Medarde A. Advanced targeted therapies in cancer: drug nanocarriers, the future of chemotherapy. Eur J Pharm Biopharm. 2015;93:52-79.

7 Chen LL. The biogenesis and emerging roles of circular RNAs. Nat Rev Mol Cell Biol. 2016; 17(4):205-11.

8 Chen LL, Yang L. Regulation of circRNA biogenesis. RNA Biol. 2015;12(4):381-8.

9 Kristensen LS, Andersen MS, Stagsted LVW, Ebbesen KK, Hansen TB, Kjems J. The biogenesis, biology and characterization of circular RNAs. Nat Rev Genet. 2019;20(11):67591.

10 Wu J, Qi X, Liu L, Hu X, Liu J, Yang J, et al. Emerging epigenetic regulation of circular RNAs in human cancer. Mol Ther Nucleic Acids. 2019;16:589-96.

11 Patop IL, Kadener S. circRNAs in cancer. Curr Opin Genet Dev. 2018;48:121-7.

12 Kristensen LS, Hansen TB, Venø MT, Kjems J. Circular RNAs in cancer: opportunities and challenges in the field. Oncogene. 2018;37(5): 555-65.

13 Huang G, Liang M, Liu H, Huang J, Li P, Wang C, et al. CircRNA hsa_circRNA_104348 promotes hepatocellular carcinoma progression through modulating miR-187-3p/ RTKN2 axis and activating $\mathrm{Wnt} / \beta$-catenin pathway. Cell Death Dis. 2020;11(12):1065.
14 Zhao Y, Hu Y, Shen Q, Chen Q, Zhu XJ, Jiang SS, et al. CircRNA_MYLK promotes malignant progression of ovarian cancer through regulating microRNA-652. Eur Rev Med Pharmacol Sci. 2020;24(10):5281-91.

15 Cen J, Liang Y, Huang Y, Pan Y, Shu G, Zheng $\mathrm{Z}$, et al. Circular RNA circSDHC serves as a sponge for miR-127-3p to promote the proliferation and metastasis of renal cell carcinoma via the CDKN3/E2F1 axis. Mol Cancer. 2021; 20(1):19.

16 Yang CY, Wang J, Zhang JQ, Cai HM. Human circular RNA hsa_circRNA_101705 (circTXNDC11) regulates renal cancer progression by regulating MAPK/ERK pathway. Bioengineered. 2021;12(1):4432-41.

17 Liang Y, Song X, Li Y, Chen B, Zhao W, Wang L, et al. LncRNA BCRT1 promotes breast cancer progression by targeting miR-1303/ PTBP3 axis. Mol Cancer. 2020;19(1):85.

18 Chen XT, Li ZW, Zhao X, Li ML, Hou PF, Chu SF, et al. Role of circular RNA in kidneyrelated diseases. Front Pharmacol. 2021;12: 615882.

19 Yang L, Zou X, Zou J, Zhang G. Functions of circular RNAs in bladder, prostate and renal cell cancer (Review). Mol Med Rep. 2021; 23(5):307.

20 Lei X, Yang M, Xiao Z, Zhang H, Tan S. circTLK1 facilitates the proliferation and metastasis of renal cell carcinoma by regulating miR-495-3p/CBL axis. Open Life Sci. 2021; 16(1):362-74

21 Gao P, Huang Y, Hou Y, Li Q, Wang H. Circular RNA ITCH is a tumor suppressor in clear cell renal cell carcinoma metastasis through miR-106b-5p/PDCD4 axis. J Immunol Res. 2021;2021:5524344.

22 Cheng L, Cao H, Xu J, Xu M, He W, Zhang W, et al. Circ_RPL23A acts as a miR-1233 sponge to suppress the progression of clear cell renal cell carcinoma by promoting ACAT2. J Bioenerg Biomembr. 2021;53(4):415-28.
23 Memczak S, Jens M, Elefsinioti A, Torti F, Krueger J, Rybak A, et al. Circular RNAs are a large class of animal RNAs with regulatory potency. Nature. 2013;495(7441):333-8.

24 Hansen TB, Kjems J, Damgaard CK. Circular RNA and miR-7 in cancer. Cancer Res. 2013 . 73(18):5609-12.

25 Bai S, Wu Y, Yan Y, Shao S, Zhang J, Liu J, et al. Construct a circRNA/miRNA/mRNA regulatory network to explore potential pathogenesis and therapy options of clear cell renal cell carcinoma. Sci Rep. 2020;10(1):13659.

26 Zeng X, Liao H, Wang F. MicroRNA-384 inhibits nasopharyngeal carcinoma growth and metastasis via binding to Smad5 and suppressing the $\mathrm{Wnt} / \beta$-catenin axis. Cytotechnology. 2021;73(2):203-15.

27 Zhong BZ, Wang Q, Liu F, He JL, Xiong Y, Cao J. Effects of miR-384 and miR-134-5p acting on YY1 signaling transduction on biological function of gastric cancer cells. Onco Targets Ther. 2020;13:9631-41.

28 Yan L, Wu K, Du F, Yin X, Guan H. miR-384 suppressed renal cell carcinoma cell proliferation and migration through targeting RAB23. J Cell Biochem. 2018.

29 Song H, Rao Y, Zhang G, Kong X. MicroRNA-384 inhibits the growth and invasion of renal cell carcinoma cells by targeting astrocyte elevated gene 1. Oncol Res. 2018;26(3): 457-66.

30 Guo L, Wang D, Zhang Z. MiR-384 represses tumorigenesis by regulating CDK6 and predicts prognosis of clear cell renal cell carcinoma. J BUON. 2018;23(3):787-94.

31 Li Q, Pan Y, Cao Z, Zhao S. Comprehensive analysis of prognostic value and immune infiltration of chromobox family members in colorectal cancer. Front Oncol. 2020;10: 582667.

$32 \mathrm{Wu} \mathrm{C}$, Zhang J. Long non-coding RNA LOXL1-AS1 sponges miR-589-5p to up-regulate CBX5 expression in renal cell carcinoma. Biosci Rep. 2020;40(11):BSR20200212. 